\title{
Posterior Medial Frontal Cortex Activity Predicts Post-Error Adaptations in Task-Related Visual and Motor Areas
}

\author{
Claudia Danielmeier, ${ }^{1}$ Tom Eichele, ${ }^{2}$ Birte U. Forstmann, ${ }^{3}$ Marc Tittgemeyer, ${ }^{1}$ and Markus Ullsperger ${ }^{1,4}$ \\ ${ }^{1}$ Max Planck Institute for Neurological Research, 50931 Cologne, Germany, ${ }^{2}$ Department of Biological and Medical Psychology, University of Bergen, 5009 \\ Bergen, Norway, ${ }^{3}$ Spinoza Center for Neuroimaging, University of Amsterdam, 1018 WS Amsterdam, the Netherlands, and ${ }^{4}$ Donders Institute for Brain, \\ Cognition and Behaviour, Radboud University, 6525 HR Nijmegen, the Netherlands
}

As Seneca the Younger put it, "To err is human, but to persist is diabolical." To prevent repetition of errors, human performance monitoring often triggers adaptations such as general slowing and/or attentional focusing. The posterior medial frontal cortex (pMFC) is assumed to monitor performance problems and to interact with other brain areas that implement the necessary adaptations. Whereas previous research showed interactions between pMFC and lateral-prefrontal regions, here we demonstrate that upon the occurrence of errors the pMFC selectively interacts with perceptual and motor regions and thereby drives attentional focusing toward task-relevant information and induces motor adaptation observed as post-error slowing. Functional magnetic resonance imaging data from an interference task reveal that error-related pMFC activity predicts the following: (1) subsequent activity enhancement in perceptual areas encoding task-relevant stimulus features; (2) activity suppression in perceptual areas encoding distracting stimulus features; and (3) post-error slowing-related activity decrease in the motor system. Additionally, diffusion-weighted imaging revealed a correlation of individual post-error slowing and white matter integrity beneath pMFC regions that are connected to the motor inhibition system, encompassing right inferior frontal gyrus and subthalamic nucleus. Thus, disturbances in task performance are remedied by functional interactions of the pMFC with multiple task-related brain regions beyond prefrontal cortex that result in a broad repertoire of adaptive processes at perceptual as well as motor levels.

\section{Introduction}

When errors are committed, it is important that adaptive processes take effect immediately to generate adequate behavioral adjustments and prevent further errors. Behavioral adjustments in post-error trials occur at motor and attentional levels. Posterror slowing (PES) (Rabbitt, 1966) is a general adjustment thought to "buy" time to enable more controlled responding (Ridderinkhof et al., 2004a). The prefrontal cortex has been implicated in top-down control on other brain areas to guide behavior (Fuster, 2000; Miller and Cohen, 2001; Miller and D'Esposito, 2005). More specifically, monitoring for errors and signaling the need for adjustments has been associated with the posterior medial frontal cortex (pMFC) (Botvinick et al., 2001; Ridderinkhof et al., 2004b). However, how performance-related signals from the pMFC modulate sensory representations is still unclear. Current theories suggest that the pMFC interacts with the lateral prefrontal cortex (LPFC), which in turn is assumed to implement the necessary top-down control (Kerns et al., 2004;

\footnotetext{
Received Aug. 17, 2010; revised Nov. 17, 2010; accepted Dec. 1, 2010.

We thank J. Derrfuß for valuable discussions on the manuscript and S. Döring, C. Selbach, K. Wittenberg, E. Bannemer, R. Rusniak-Florath, and P. Euskirchen for help with data collection. T.E. was supported through a NEVRONOR grant from the Norwegian Research Council. B.U.F. is supported by a Netherlands Organization for Scientific Research (NWO) Veni grant.

Correspondence should be addressed to Claudia Danielmeier, Max Planck Institute for Neurological Research, Cognitive Neurology Research Group, Gleueler Straße 50, 50931 Cologne, Germany. E-mail: c.danielmeier@ donder.ru.nl.

DOI:10.1523/JNEUROSCI.4299-10.2011

Copyright $\odot 2011$ the authors $\quad 0270-6474 / 11 / 311780-10 \$ 15.00 / 0$
}

Carter and van Veen, 2007). Here, we investigate whether errorrelated activity in the pMFC directly predicts post-error adaptations in motor and perceptual systems in an interference task.

Recent studies suggest that successful implementation of control upon conflict is reflected in visual areas that are crucial for solving the task at hand (Egner and Hirsch, 2005; Wylie et al., 2006). In a task-switching paradigm, Wylie et al. (2006) found activity enhancement in the task-relevant visual brain area but no indicators for suppressed activity in task-irrelevant areas. However, suppression of neuronal activity due to attentional mechanisms has been observed in striate and extrastriate visual areas in monkeys (Ferrera and Lisberger, 1997; Vanduffel et al., 2000; Treue, 2001) and humans (Müller and Kleinschmidt, 2004; Baier et al., 2006).

We hypothesized that error-related pMFC activity predicts both subsequent signal increases in perceptual areas encoding task-relevant stimulus features and signal decreases in perceptual areas encoding distracting stimulus features. Additionally, we expected PES to be related to preceding error-related pMFC activity (Garavan et al., 2002; Kerns et al., 2004; Debener et al., 2005) and to activity changes in the motor system. Since structural correlates of PES are yet unknown, we calculated correlations between diffusion-weighted imaging (DWI) and PES values. PES has been suggested to result from an increased motor threshold (Botvinick et al., 2001) that might be implemented by general motor inhibition. Therefore, we expected the structural analysis to reveal a network connecting brain regions implicated in motor inhibition, in particular right-hemispheric pre-supplementary area, in- 
ferior frontal cortex, and subthalamic nucleus (STN) (Aron et al., 2007).

Miller and D'Esposito (2005) have pointed out that the temporal overlap of the hemodynamic response in successive trials renders it difficult to investigate spatiotemporal dynamics inherent in top-down processes with standard functional magnetic resonance imaging (fMRI) analysis. This is especially problematic when two trials of interest, e.g., error and post-error trials, occur always in the same order by definition. Therefore, a group-level spatial independent component analysis (ICA) (Calhoun et al., 2001) was used to decompose the data into separate independent components (ICs), followed by deconvolution to extract the trialby-trial dynamics of sequential effects. To study error-driven adjustments in cognitive control, we investigated ICs showing significant activity changes in error and post-error trials.

\section{Materials and Methods}

\section{Participants}

Twenty-one neurologically and psychiatrically healthy participants took part in this study. One male participant had to be excluded from data analysis because he did not complete the task. Thus, the final sample consisted of 11 female and 9 male participants (mean age: 24.1 years; range: $21-35$ years of age). Participants were all right handed and showed no signs of color vision deficiencies. Before fMRI measurements, participants provided written informed consent. The study was performed according to the Declaration of Helsinki.

\section{Behavioral task}

Participants performed a moving-dots interference task similar to a Simon task (Simon, 1969) in the scanner. On each trial, a cloud of colored moving dots (coherently moving leftward or rightward and extending across $4.3^{\circ}$ of visual angle) was presented centrally on a computer screen. Speed of the moving dots was 10.2 degrees of visual angle per second. Participants were asked to indicate the color of the dots (relevant feature) by pressing a button with their left or right index finger while ignoring the motion direction (irrelevant feature). Four isoluminant colors were chosen from the Teufel colors (Teufel and Wehrhahn, 2000). The colors light blue and beige required a left button press, while turquoise and light pink required a right button press. Motion direction could either be congruent or incongruent with the required response side. The proportion of congruent and incongruent trials was 50\% each. The trial sequence was pseudo-randomized to avoid direct repetitions of the same color in two consecutive trials and to counterbalance the transitions of congruent and incongruent trials. Trial duration was $5 \mathrm{~s}$ on average. To improve temporal sampling of the hemodynamic response (HR), trials were "jittered" with onset delays of $0,330,660,1000,1330$, or $1660 \mathrm{~ms}$, resulting in an oversampling of the actual image acquisition time of $2 \mathrm{~s}$ by a factor of 6 . After this variable onset delay, during which a central fixation cross was displayed on the screen, colored moving dots were presented until a response was given, but maximally for $1500 \mathrm{~ms}$. Finally, a fixation cross appeared on the screen until the total trial duration reached $5 \mathrm{~s}$. The experiment consisted of 336 experimental trials and 40 null-events pseudo-randomly interspersed in the experimental trials to affect all transition types equally. Performance feedback was presented before every other null event for a duration of $2 \mathrm{~s}$. Participants were informed about the amount of correct and erroneous responses since the last feedback. If the participant's response was slower than an individually adapting response deadline on more than three trials since the last feedback, the feedback showed an additional "too slow" message. The response deadline for the calculation of slow responses was initially set to $1000 \mathrm{~ms}$ and subsequently adapted according to the subject's average error rate in incompatible trials.

\section{Functional localizer}

In addition to the task described above, we ran two functional localizer tasks after acquisition of the Simon task to identify the color and motion processing visual areas in each participant. The order of color and motion localizer was counterbalanced across participants.
The color localizer consisted of 12 blocks where (stationary) colored dots were presented in the center of the screen and 13 blocks with gray dots. Colored and gray dots were approximately isoluminant. Each image was shown for $700 \mathrm{~ms}$, followed by $300 \mathrm{~ms}$ of blank screen. Each block consisted of 15 images, and thus one block lasted for $15 \mathrm{~s}$. In colored images, two colors were combined in one image: $50 \%$ of the dots were presented in one color and $50 \%$ in another color. Colors were the same as in the Simon task. In rare catch trials, all dots were presented in the same color. Participants had to indicate this with a button press. Catch trials were introduced to keep participants focused on the colors. They occurred only six times, pseudo-randomly spread across the experiment.

The motion localizer also consisted of 12 stimulation blocks and 13 rest blocks. In stimulation blocks, 15 trials of gray moving dots were presented. In each trial, dots were presented for $500 \mathrm{~ms}$ followed by a fixation cross, which was also presented for $500 \mathrm{~ms}$. Speed of the moving dots was $9^{\circ}$ of visual angle per second. In rare catch trials, dots moved noticeably faster with $24^{\circ}$ per second. To keep participants focused on the motion, they had to indicate the presence of a catch trial with a button press. Six catch trials were presented in the course of this experiment. During rest blocks, stationary gray dots were presented for $15 \mathrm{~s}$.

\section{Image acquisition: $\mathrm{fMRI}$ and anatomical data}

Data acquisition was performed on a $3 \mathrm{~T}$ Siemens Trio scanner. Thirty slices ( $3 \mathrm{~mm}$ thickness, $3 \times 3 \times 3 \mathrm{~mm}$ voxel size, $0.3 \mathrm{~mm}$ interslice gap) were obtained in an interleaved fashion parallel to the anterior commissure-posterior commissure line using a single-shot gradient echo-planar imaging (EPI) sequence [repetition time (TR): $2000 \mathrm{~ms}$; echo time (TE): $30 \mathrm{~ms}$; bandwidth: $116 \mathrm{kHz}$; flip angle: $90^{\circ}$; $64 \times 64$ pixel matrix; field of view: $192 \mathrm{~mm}$ ]. Before functional scanning, a high resolution $(1 \times 1 \times$ $1.25 \mathrm{~mm}$ ) anatomical brain image was recorded from each participant in a separate session using a modified driven equilibrium Fourier transform sequence (TR: $1930 \mathrm{~ms}$; TE: $5.8 \mathrm{~ms}$ ).

\section{Image acquisition: diffusion-weighted data}

Diffusion-weighted images were acquired in a separate scanning session using a twice-refocused spin-echo echo-planar imaging (TR: $12 \mathrm{~s}$; TE: $100 \mathrm{~ms} ; 72$ axial slices; resolution: $1.72 \times 1.72 \times 1.7 \mathrm{~mm}$ ). We used a GRAPPA (generalized autocalibrating partially parallel acquisition) technique (reduction factor: 2.0) for parallel imaging. Diffusion weighting was isotropically distributed along 60 directions with a $b$ value of 1000 $\mathrm{s} / \mathrm{mm}^{2}$. The high angular resolution of the diffusion weighting directions improves the robustness of probability density estimation by increasing the signal-to-noise ratio and reducing directional bias. Additionally, seven datasets with no diffusion weighting (b0) and a $b$ value of 500 $\mathrm{s} / \mathrm{mm}^{2}$ were acquired initially and interleaved after each block of 10 diffusion-weighted images: Here the b0 images serve as anatomical reference for motion correction, and the b500 images serve to suppress pseudo-diffusion and cope with the CSF contamination effect. To further increase signal to noise, we acquired three consecutive scans that were subsequently averaged together. The entire data acquisition protocol lasted $\sim 45 \mathrm{~min}$.

\section{Image processing and analysis: localizer tasks}

Analysis of fMRI data of the functional localizer tasks was carried out using the Oxford Centre for Functional MRI of the Brain (FMRIB) Software Library (FSL), version 4.0.3 (http://www.fmrib.ox.ac.uk/fsl) (Smith et al., 2004). Non-brain data were removed from functional and anatomical datasets using the Brain Extraction Tool (BET) (Smith, 2002). Functional data were motion corrected using rigid body registration to the central volume (Jenkinson et al., 2002) and corrected for slice acquisition time differences using sinc interpolation. Low-frequency signals were removed using a $1 / 30 \mathrm{~Hz}$ highpass filter. Spatial smoothing was applied using a Gaussian filter with $5 \mathrm{~mm}$ full width at half maximum (FWHM). Registration of the EPI images with the high resolution brain images and normalization into standard [Montreal Neurological Institute (MNI)] space was carried out using affine registration (Jenkinson and Smith, 2001). A general linear model was fitted into prewhitened data space to account for local autocorrelations (Woolrich et al., 2001). 
FMRI image processing and analysis of interference task

Preprocessing. All images were realigned to the first image in the time series to correct for head movement and then normalized to the MNI reference space using SPM5 statistical parametric mapping (http://www. fil.ion.ucl.ac.uk/spm). Normalized data were resliced to a cubic voxel size of $3 \mathrm{~mm}^{3}$ and smoothed with a Gaussian kernel with $8 \mathrm{~mm}$ FWHM.

Independent component analysis. As pointed out above, it is difficult to analyze the temporal dynamics of successive trials with standard fMRI analysis procedures. Therefore, here we employed a group-level ICA followed by deconvolution of the HR as described by Eichele et al. (2008). In brief, the ICA analysis was followed by estimation of subject- and IC-specific HRs. The HRs were deconvolved from the time courses of the ICs to recover empirical HRs (Aguirre et al., 1998; Handwerker et al., 2004). These were used subsequently for estimation of the amplitude of the event-related response to each trial (Eichele et al., 2008). For an overview of the ICA and subsequent statistical analyses, see the flow chart in supplemental Figure 1 (available at www.jneurosci.org as supplemental material).

Error monitoring and the various subsequent adaptations are likely implemented by different brain networks (pMFC, motor system, visual perceptual areas), such that we expected these networks to be reflected in separate ICs. We first decomposed the data using the group spatial ICA rationale proposed by Calhoun et al. (2001), which is implemented in GIFT (Group ICA of fMRI Toolbox; http://Mialab.mrn.org/software/ index.php), running in MATLAB. For each individual separately, the preprocessed fMRI data were prewhitened and reduced via temporal principal component analysis (PCA) to 100 components. Then, grouplevel aggregate data were generated by concatenating and reducing individual principal components in a second PCA step. Infomax ICA was performed in this set with a high model order of 100 components (Kiviniemi et al., 2009). To estimate robust components, the decomposition was performed 100 times with random initial conditions and identified centroids with a canonical correlation-based clustering (ICASSO; Himberg et al., 2004). All components that we interpret in this dataset have a robustness index of higher than 0.9. Individual IC maps and time courses were back-reconstructed by multiplying the corresponding data with the respective portions of the estimated demixing matrix. The group average maps were inspected to identify and discard those ICs primarily associated with artifacts representing signal from large vessels, ventricles, motion, and susceptibility. From the remaining ICs, only those with significant random effects $t$ statistics of their maps set at a threshold of $t_{(19)}>5$ [uncorrected $p=4 \cdot 10^{-5}$; this uncorrected $t$ threshold being equal or exceeding the threshold estimated for a false discovery rate (FDR) corrected $p<0.05$ in any of the maps, since FDR varies across maps as a function of the distribution of the $p$ values] and cluster extent of at least 27 contiguous voxels were considered further. In the time courses of these ICs, we focused on the frequency range relevant for event-related hemodynamic responses and filtered the individual time courses with a $64 \mathrm{~s}$ Butterworth high-pass filter.

Deconvolution. For this analysis step we used the method reported in Eichele et al. (2008). For each participant and component separately, the empirical event-related HRs were deconvolved by forming the convolution matrix of all trial onsets with a length of $20 \mathrm{~s}$ and multiplying the pseudoinverse of this matrix with the filtered and normalized IC time course. Estimation of single-trial amplitudes was performed in 26 of 100 ICs where consistent event-related activations, i.e., significant positive peaks in the HR, were present in the time range of 3-12 s. In this set, single trial amplitudes were recovered by fitting a design matrix containing separate predictors for each trial onset convolved with the estimated HR onto the IC time course, estimating the scaling coefficients $(\beta)$ by using multiple linear regression.

Inference. We then removed variability associated with factors of no interest from the data by predicting the respective single trial amplitudes of each subject with a multiple linear regression design, including categorical predictors coding for the trial type (compatible, incompatible), motion direction of the moving dots (left, right), and hand response side (left, right). The last step in component selection was to identify the components with significant effects in error and/or post-error trials. The residualized data were used to estimate the amplitude modulation from four trials before and four trials after errors. Individual trial-to-trial amplitudes were entered into one-sample, two-tailed $t$ tests under the assumption of zero magnitude. Effects were considered significant at $p<$ 0.05 . Those ICs that showed significant effects in error or post-error trials were selected for correlational analyses to investigate trial-by-trial effects between them. The relationship between different ICs was investigated by correlating the IC amplitudes in error trials from one component with the slope of IC amplitudes (representing adaptation effects) in another component on a between-subjects level.

\section{DTI data analysis}

Motion correction for the diffusion-weighted images was applied to all images using seven-parameter global rescale registration (Jenkinson et al., 2002) as implemented in FSL. All baseline b0 images were aligned to a reference b0 image, and the resulting linear transformation matrices were then applied to the diffusion-weighted images following each baseline b0 image. The gradient direction for each volume was corrected using the rotation parameters. Then, the three scan repetitions were averaged to improve the signal-to-noise-ratio.

Fractional anisotropy. Fractional anisotropy (FA) images were created by fitting a tensor model to the diffusion data using FDT (FMRIB Diffusion Toolbox). Then, images were brain-extracted using BET. The following steps were done using TBSS (Tract-Based Spatial Statistics) (Smith et al., 2006): FA images of all subjects were first aligned into a standard brain space using nonlinear registration. Next, the mean of all subjects' aligned FA images was created and then "thinned" using standard image processing techniques to create a mean FA skeleton that represents the centers of major tracts common to the group of subjects. Each subject's aligned FA data were then projected (perpendicular to the local tract direction) onto this skeleton so that the projected FA values were taken from the centers of the tracts in the original FA image. This projection aimed to resolve any residual correspondence (alignment) problems after the initial nonlinear registration. The resulting data are then fed into voxelwise cross-subject statistics (Smith et al., 2007).

Because we were interested in the structural correlates of post-error adaptation effects, we computed the individual PES values as a measure of behavioral adaptation. PES values were calculated as mean post-error reaction time (RT) minus mean post-correct RT, with post-correct trials being correctly performed trials preceded by at least two and followed by at least one other correct trial.

Individual PES values were then correlated with the participants' FA values. The significance of this regression was determined by means of permutation testing (using Randomise version 2.1 implemented in FSL). Activations were considered significant at a value of $p<0.001$ with a minimum cluster size of 40 contiguous voxels.

Probabilistic tractography. Estimation of tracts was done by probabilistic tractography using published methods in the FSL environment (Behrens et al., 2007). Fiber tracking was done probabilistically, using 5000 tract-following samples at each voxel. We used a dual-fiber model as implemented in bedpostx in FDT version 2.0. This model helps to account for issues related to crossing fibers and produces more reliable results compared with a single-fiber model. The result is a brain image in which all voxels have a value that represents the connectivity (number of fibers from the probabilistic analysis) between that voxel and the voxels in the seed region. One advantage of the probabilistic tractography is that it accounts for uncertainty inherent in local fiber directions and thus estimates a spatial probability distribution of connectivity from the seed regions. Those WM regions showing significant correlations between FA values and PES were taken as seed regions for tractography. All tractography was done in each participant's native space (unnormalized) data, and the resulting maps were warped into standard space (using the MNI $1 \mathrm{~mm}$ isotropic brain as reference) for crosssubject averaging and comparison.

\section{Results}

\section{Behavioral data}

Total error rate was 5.18\% (SEM: 0.8\%). Error rates did not differ between congruent and incongruent trials $(p=0.62)$. Probability for committing errors was significantly reduced on post-error 


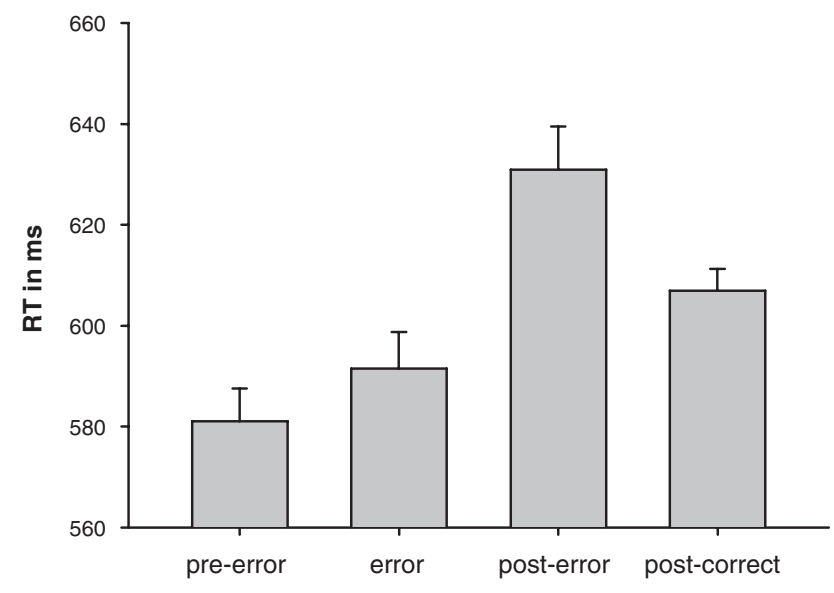

Figure 1. Mean reaction times and within-subject SEMs for pre-error, error, and post-error trials as well as for correct trials following other correct trials (post-correct). There is a significant PES effect that is defined as the difference between post-error RTs and post-correct RTs.

\begin{tabular}{ll} 
Table 1. Behavioral results & \\
\hline Trial type & Mean RT in ms (SEM) \\
\hline Correct trials & \\
CC & $603(16)$ \\
iC & $607(15)$ \\
cl & $616(18)$ \\
il & $601(18)$ \\
Trials around errors & \\
Pre-error & $581(16)$ \\
Error & $592(18)$ \\
Post-error & $631(16)$ \\
Post-correct & $607(17)$
\end{tabular}

Shown are reaction times for correct trials according to present and previous trial type ( $\mathrm{CC}$, present and previous trial both compatible; iC, previous trial incompatible, present trial compatible; cl, previous trial compatible, current trial incompatible; il, present and previous trial both incompatible) and for error trials and trials surrounding errors.

trials compared with post-correct trials $\left(2.78 \%\right.$ vs $5.25 \% ; t_{(19)}=$ $2.7, p=0.014)$, demonstrating improved accuracy as a result of post-error adjustments.

Reaction times for compatible and incompatible trials in general were similar (mean RT in congruent trials: 613 ms; RT in incongruent trials: $614 \mathrm{~ms}$ ), but a conflict adaptation effect was found (interaction "current trial congruency" $\times$ "previous trial congruency": $\left.\left.F_{(1,19}\right)=8.21 ; p=0.01\right)$, indicating an influence of previous congruency on the current RT. For further analyses trials were categorized into pre-error, error, post-error, and postcorrect trials (only correct trials preceded by at least two and followed by at least one other correct trial). A repeated-measures ANOVA showed a significant effect of trial type in RTs, $F_{(3,17)}=$ 4.86, $p=0.013$ (Fig. 1). The comparison of post-error RTs with post-correct RTs revealed post-error slowing $\left(t_{(19)}=2.3, p=\right.$ $0.033)$. PES values did not correlate with the individuals' overall RT $(r=-0.357, p=0.123)$. Behavioral results are also shown in Table 1.

\section{Error-related and post-error modulations in brain activity}

ICs showing activity modulations only in error trials

Three ICs showed modulations specifically in error trials: IC 01 covered large parts of the pMFC and additional areas associated with error processing (Ullsperger and von Cramon, 2001; Klein et al., 2007) (Fig. 2A; Table 2). Its activity was increased in error trials $\left(t_{(1,19)}=2.68, p=0.015\right)$. Furthermore, IC 02 covering the cuneus showed increased activity during errors $\left(t_{(1,19)}=2.21\right.$, $p=0.040)$, and IC 03, covering the right inferior occipital cortex and cerebellum, showed an activation decrease $\left(t_{(1,19)}=-2.18\right.$, $p=0.042$ ).

ICs showing activity modulations in both error and post-error trials

IC 04, located in the task-relevant color-encoding visual area, and IC 05, covering the task-irrelevant motion-encoding visual area (encoding the distracting stimulus dimension), were modulated in error and post-error trials. Both ICs showed a clear overlap with activations in the color and motion localizer, respectively (Fig. $2 A$ ). In the task-relevant area, activity decreased already in pre-error trials $\left(t_{(1,19)}=-2.22, p=0.039\right)$ and was further decreased in error trials $\left(t_{(1,19)}=-4.35, p<0.001\right)$. In post-error trials, activity of IC 04 increased again compared with error trials but was still below baseline $\left(t_{(1,19)}=-4.49, p=0.001\right)$ and returned to baseline within two trials following the error (Fig. $2 \mathrm{~A}$, see mean component activation time course across pre-error, error, and post-error trials).

In line with the assumption that at least some errors result from increased processing of task-irrelevant visual features, there was a trend for pre-error activity enhancement in the motionprocessing IC $05\left(t_{(1,19)}=1.64, p=0.059\right.$ in trial 2 before the error, and $t_{(1,19)}=1.37, p=0.093$ in trial 1 before the error, both one-tailed). Activity in this component then decreased in error trials $\left(t_{(1,19)}=-2.73, p=0.013\right)$, potentially reflecting the onset of adaptive processes that are continued in post-error trials. The trial immediately following an error showed further decreased activity $\left(t_{(1,19)}=-3.17, p=0.005\right)$ in these motion-processing areas, and activity was also still below baseline $\left(_{(1,19)}=-2.53\right.$, $p=0.021)$ in trial 2 after the error.

ICs showing activity modulations only in post-error trials IC 06, located in frontopolar cortex and anterior dorsolateral prefrontal cortex (DLPFC) areas (Fig. 2B), selectively showed increased amplitudes in activity in post-error trials $\left(t_{(1,19)}=2.69\right.$, $p=0.015)$. Besides the peak activation in DLPFC, this component also comprised activity in the rostral cingulate zone.

A decrease in activity was observed in two ICs showing activations in the left and right hemispheric motor system (IC 07 and IC 08, respectively). Although only the dominant left-sided IC 07 was significantly different from baseline in post-error trials $\left(t_{(1,19)}=-2.74, p=0.013\right)$, IC 08 showed a similar decrease in post-error trials as that of IC 07 , and activated brain areas in IC 08 mirrored the activations of IC 07 in the other hemisphere. To take both left and right hemispheric motor activations into account in the following correlational analysis, we therefore averaged amplitudes from IC 07 and IC 08. This averaged motor IC shows a significant decrease in post-error trials compared with error trials $\left(t_{(1,19)}=-2.40, p=0.027\right)$.

\section{Correlations between frontal and visual components}

Two ICs were located in frontal cortical areas that, according to current theories (Botvinick et al., 2004; Kerns et al., 2004; Carter and van Veen, 2007), could be the source of top-down modulations in visual areas: the monitoring-related pMFC-centered IC 01, modulated in error trials, and the DLPFC/frontopolar IC 06, modulated in post-error trials. To investigate whether the pMFC component is related to performance monitoring and initiates an increase in activity in the lateral prefrontal cortex that then in turn regulates top-down modulations, or whether the pMFC shows a direct functional link to modulations in visual areas, we conducted correlations between both IC 01 and IC 06 on the one hand and activation changes in color-encoding areas (IC 04, en- 
coding relevant stimulus dimension) and motion-encoding areas (IC 05, encoding distracting stimulus dimension) on the other hand. Adaptation effects in IC 04 were quantified as the slope of activity between error and post-error trials (Fig. 2). As indicated by the mean activation plot of this component, activity in colorencoding areas was lowest in the error trial and increased again in the following trials, presumably reflecting adaptive mechanisms. In contrast, in motion-related areas activity is already reduced in error trials (compared with pre-error) and further decreases in post-error trials. This seems to indicate that adaptive mechanisms in motion-processing areas occur earlier than in color-processing areas and affect the activation level in the error trial already. Therefore, adaptive effects in motion areas were quantified as the slope of activity between pre-error and post-error trials.

Greater error-related activity in the pMFC-centered IC 01 predicted a stronger post-error signal increase in colorencoding areas $(r=0.59, p=0.006$, Cook's distance of all values $<1$ ) (Fig. 3 ). Moreover, error-related IC 01 activity was negatively correlated with the activity change in motion-processing areas $(r=$ $-0.56, p=0.011$; Cook's distance of all values $<1)$ : The more pMFC activity in the error trial, the stronger the decrease in motion areas from pre-error to post-error trials. This suggests that pMFC activity triggers both enhancement in the visual area relevant for encoding the stimulus (color) and inhibition of the area responsible for encoding the distracting dimension (motion).

In contrast, the enhanced post-error DLPFC activity (IC 06) was neither correlated with the activation increase in color areas $(p=0.28)$ nor with the activation decrease in motion areas $(p>$ $0.34)$. Furthermore, in contrast to what would have been predicted according to Kerns et al. (2004), pMFC activity in error trials did not correlate with DLPFC activity in post-error trials $(p=0.16)$. Neither the pMFC component (in the error trial) nor the dorsolateral/frontopolar component (post-error) were correlated with the post-error activation change in the right lateralized inferior occipital component (IC 03, both $p>0.18$ ).

\section{Correlations between frontal and motor components}

Adaptive processes in the mean motor IC were quantified as slope of decreasing activity between error and post-error trials. Strength of activity in pMFC (IC 01) predicted the amount of activation decrease in the mean motor IC $(r=-0.74, p<0.001$; Cook's distance of all values $<1$ ). The larger pMFC activity in error trials, the stronger the activation decrease in motor areas in post-error trials.

Furthermore, the level of motor activation in the post-error trial was negatively correlated with the behavioral measure of post-error slowing $(r=-0.46, p=0.043$; for one value Cook's distance was 1.013; after removing this case the correlation remained significant, $r=-0.54, p=0.017)$. Lower post-error activity in the motor ICs was associated with greater post-error slowing.

\section{Diffusion imaging results}

Correlations between fractional anisotropy values and participants' post-error slowing measures were computed, to identify those white matter regions where individual differences in FA contribute to different behavioral outcomes. Note, that at least parts of the correlating WM tracts might serve inhibitory functions. This assumption is due to the fact that the FA values of interest correlate with prolonged reaction times, i.e., PES. FA in two medial frontal WM regions correlated with PES (Fig. 4a; Table 3): the left anterior midcingulate region and the right presupplementary motor area (pre-SMA).

These two WM areas were taken as seed points for probabilistic tractographies to reveal those brain regions that are connected by the PES-related fiber tracts. The tractography from the preSMA seed region showed a network that has previously been described as an inhibitory network related to the slowing or stopping of motor responses (Aron et al., 2007). This tract comprises the pre-SMA, posterior inferior frontolateral areas, and the STN of the right hemisphere (Fig. $4 b$ ). Parts of this tractography reached the left-hemispheric pMFC, but overall the network was mainly located within the right hemisphere.

WM tracts, running through the anterior midcingulate seed region, connect frontal dorsolateral and frontopolar areas with the medial frontal cortex. Moreover, there seem to be connec- 
Table 2. MNI coordinates, maximum $t$ value, and volume of activated areas of independent components showing significant modulations in error and/or post-error trials

\begin{tabular}{|c|c|c|c|c|c|c|}
\hline $\mathrm{IC}$ & Region & Volume $\left(\mathrm{mm}^{3}\right)$ & Maximum $t$ value & $x$ & $y$ & $z$ \\
\hline \multirow[t]{15}{*}{ IC 01 (modulation in error trials) } & \multirow[t]{5}{*}{ Posterior medial frontal cortex } & \multirow[t]{5}{*}{36,999} & 33.73 & 3 & -1 & 48 \\
\hline & & & 24.13 & -1 & 7 & 32 \\
\hline & & & 20.57 & 7 & 11 & 30 \\
\hline & & & 16.77 & 13 & 11 & 47 \\
\hline & & & 15.92 & 2 & 17 & 43 \\
\hline & \multirow[t]{2}{*}{ Right operculum/insula } & \multirow[t]{2}{*}{6790} & 11.20 & 53 & 8 & -5 \\
\hline & & & 9.17 & 53 & -22 & 17 \\
\hline & Left posterior insula & 3930 & 11.09 & -36 & -20 & 11 \\
\hline & Left anterior insula & 911 & 6.82 & -32 & 8 & 4 \\
\hline & Left premotor cortex & 4438 & 13.25 & -39 & -14 & 41 \\
\hline & Left posterior SFS & 283 & 7.04 & -26 & -7 & 64 \\
\hline & Right premotor cortex & 348 & 6.20 & 45 & -14 & 41 \\
\hline & Posterior MTG & 968 & 6.86 & -54 & -56 & 23 \\
\hline & Left inferior parietal lobule & 237 & 5.99 & -50 & -65 & 32 \\
\hline & Left hypothalamus & 451 & 8.78 & -7 & -17 & -1 \\
\hline \multirow[t]{3}{*}{ IC 02 (modulation in error trials) } & \multirow[t]{3}{*}{ Cuneus } & \multirow[t]{3}{*}{69,611} & 27.89 & 0 & -71 & 18 \\
\hline & & & 27.35 & -6 & -68 & 10 \\
\hline & & & 22.31 & -7 & -61 & -3 \\
\hline \multirow[t]{5}{*}{ IC 03 (modulation in error trials) } & \multirow[t]{4}{*}{ Right inferior occipital cortex/cerebellum } & \multirow[t]{4}{*}{24,359} & 14.05 & 35 & -74 & -24 \\
\hline & & & 13.89 & 47 & -70 & -16 \\
\hline & & & 11.83 & 26 & -89 & -17 \\
\hline & & & 9.76 & 47 & -71 & -11 \\
\hline & Left posterior SFG & 439 & 7.40 & -26 & 1 & 64 \\
\hline \multirow[t]{7}{*}{ IC 04 (modulation in error and post-error trials) } & \multirow[t]{3}{*}{ Left color-encoding area } & \multirow[t]{3}{*}{29,710} & 23.72 & -25 & -86 & -17 \\
\hline & & & 19.05 & -34 & -80 & -11 \\
\hline & & & 18.88 & -11 & -97 & -13 \\
\hline & Right color-encoding area & 15,577 & 15.81 & 18 & -97 & -3 \\
\hline & & & 15.64 & 33 & -94 & -9 \\
\hline & & & 13.43 & 28 & -85 & 9 \\
\hline & Left parahippocampal gyrus & 444 & 9.14 & -17 & -26 & -11 \\
\hline IC 05 (modulation in error and post-error trials) & Right motion-encoding area (and surrounding areas) & 73,682 & 27.12 & 53 & -70 & -1 \\
\hline & & & 22.13 & 47 & -61 & -12 \\
\hline & & & 19.40 & 53 & -74 & -1 \\
\hline & & & 19.07 & 44 & -70 & -16 \\
\hline & & & 13.60 & 30 & -79 & 21 \\
\hline & Left motion-encoding area & 12,684 & 17.09 & -36 & -80 & 2 \\
\hline & & & 14.61 & -42 & -68 & -2 \\
\hline & & & 12.12 & -46 & -65 & 2 \\
\hline & Caudate nucleus & 177 & 5.66 & 19 & 16 & 9 \\
\hline IC 06 (modulation in post-error trials) & Frontopolar cortex/anterior dorsolateral cortex & 49,272 & 25.37 & 38 & 53 & 17 \\
\hline & & & 20.08 & 22 & 64 & 23 \\
\hline & & & 16.23 & -22 & 61 & 3 \\
\hline & & & 15.39 & 5 & 64 & 29 \\
\hline & Rostral cingulate zone & 2646 & 7.69 & -4 & 31 & 22 \\
\hline & Collateral sulcus & 258 & 6.48 & 26 & -8 & -33 \\
\hline & Left cerebellum & 458 & 7.06 & -38 & -59 & -34 \\
\hline IC 07 (modulation in post-error trials) & Left motor cortex & 31,145 & 35.96 & -36 & -29 & 53 \\
\hline & & & 23.69 & -43 & -25 & 46 \\
\hline & & & 16.25 & -27 & -44 & 59 \\
\hline & Left posterior insula & 5876 & 12.98 & -46 & -14 & 11 \\
\hline & SMA & 3558 & 9.56 & -3 & -16 & 43 \\
\hline & Left thalamus & 592 & 9.60 & -13 & -20 & -1 \\
\hline & Right cerebellum & 5562 & 14.66 & 23 & -46 & -29 \\
\hline IC 08 (modulation in post-error trials) & Right motor cortex & 35,271 & 44.91 & 48 & -22 & 50 \\
\hline & & & 28.85 & 42 & -23 & 57 \\
\hline & & & 24.36 & 45 & -32 & 50 \\
\hline & & & 17.16 & 53 & -11 & 53 \\
\hline & Right posterior insula & 6674 & 13.81 & 44 & -16 & 11 \\
\hline & SMA & 2117 & 12.97 & 10 & -19 & 45 \\
\hline & Left postcentral gyrus & 1035 & 7.33 & -33 & -32 & 55 \\
\hline & Right thalamus & 328 & 8.58 & 17 & -19 & -1 \\
\hline & Left central sulcus & 201 & 6.67 & -48 & -26 & 44 \\
\hline & Left cerebellum & 5236 & 12.61 & -11 & -50 & -30 \\
\hline
\end{tabular}

IC 01 shows an extended activation in the posterior medial frontal cortex. IC 02 shows one large activation in the cuneus, and the activation of IC 03 is mainly located in the right inferior occipital cortex, extending into the cerebellum. Activations in IC 04 correspond to color-processing visual areas, and activations in IC 05 correspond to motion-processing visual areas. IC 06 shows a post-error activation increase predominantly in frontopolar and anterior dorsolatera prefrontal areas. IC 07 and IC 08 reflect activations in the left and right motor system, respectively. All listed activations have a minimum volume size of $135 \mathrm{~mm}^{3}$ (five contiguous voxels). SFG, Superior frontal gyrus; SFS, superior frontal sulcus; MTG, middle temporal gyrus; SMA, supplementary motor area. 
tions between medial frontal cortex and posterior running tracts, with the latter probably representing parts of the inferior fronto-occipital fascicle. There were also projections toward inferior thalamic nuclei and toward anterior cingulate regions in the contralateral hemisphere. However, these tracts were mainly located within the left hemisphere. Tracts from both preSMA and anterior midcingulate seed regions extended to WM regions beneath the pMFC component (IC 01), suggesting a link between cortical and WM areas associated with PES.

\section{Discussion}

The present experiment was set up to investigate the behavioral and neuronal level of post-error adaptations on a trialto-trial basis, as well as the structural connections supporting these functions. It is assumed that post-error adaptations are triggered by the performance monitoring system and serve to improve future performance, i.e., avoid further errors. Participants indeed showed improved accuracy and prolonged reaction times in post-error trials (PES).

Seven ICs showed activity modulations in error and post-error trials. The IC showing selectively enhanced activity dur-

ing error trials comprised regions usually implicated in performance monitoring (Ullsperger and von Cramon, 2001; Ridderinkhof et al., 2004b): the peak activity of this monitoring IC was located in the pMFC; smaller coactivations were located in the anterior insula bilaterally. The monitoring IC showed the most relevant result to our inquiry, since its level of activity in error trials predicted the strength of peri-error and post-error adjustments in task-related visual ICs and in the motor system. As the time course is equal for all parts of an IC, it is possible that, in addition to $\mathrm{pMFC}$, anterior insula activity contributes to driving these adaptive changes.

Activity in task-relevant color-encoding areas was low in error trials but showed an increase in post-error trials, whereas the taskirrelevant, motion-processing IC (encoding distracters) decreased after errors. This decrease already started during the error trial and continued in the post-error phase. Both color area increase and motion area decrease were correlated with the preceding level of errorrelated pMFC activity. These changes in post-error activity may help to prevent further errors by enhancing activity in relevant brain areas and inhibiting distracting information at the same time. The result corroborates the suggestion that the pMFC both detects performance problems and acts to change behavior (Ridderinkhof et al., 2004b; Kennerley et al., 2006). Previously, only links between pMFC function and motor-related adaptations (PES, response selection) have been shown (Debener et al., 2005). Our data not only support those previous findings but provide evidence for an important extension of pMFC function: it appears to act on task-related visual areas as well. Sarter et al. (2006) hypothesized that anterior cingulate cholinergic activity contributes (presumably via the basal forebrain) to the recruitment of top-down control mainly to attenuate effects of distracting input, but they speculated that evidence for suppression of task- irrelevant cortical regions has remained scarce due to methodological reasons. However, suppression of striate and extrastriate neuronal activity has been observed before in macaques (Ferrera and Lisberger, 1997; Vanduffel et al., 2000; Treue, 2001). Thus, inhibitory effects in motion areas seem to be plausible in the present task, where motion direction is the distracting stimulus dimension.

Egner and Hirsch (2005) dissociated activity changes in the fusiform face area (FFA) when it was task relevant from changes in the same area when it was task irrelevant. By using a name-face interference task, they demonstrated that the activation in FFA can be modulated by an interaction of cognitive control and conflict. While they found a modulation when FFA was the taskrelevant visual area, they did not find any modulation when FFA was the irrelevant visual area. These FFA modulations were observed in correct trials that were influenced differentially by conflict. They did not report a functional relationship between pMFC areas and adaptations in FFA, but instead suggested that the LPFC drives the changes in FFA.

Based on previous studies, one might expect a close interaction between the pMFC and DLFPC, which has been associated with the implementation of cognitive control (Garavan et al., 2002; Botvinick et al., 2004; Kerns et al., 2004; Cavanagh et al., 2009). Although we did find a DLPFC component showing posterror activation enhancement, this IC did not correlate with either the pMFC or the color and motion ICs. This corresponds with results by Hyafil et al. (2009) and Kouneiher et al. (2009) that also did not show any cognitive control-specific correlation between pMFC and DLPFC activity. Thus, at least in some tasks pMFC seems to be more directly linked to adjustment processes in stimulus-encoding areas than DLPFC. It has been suggested that lateral prefrontal areas maintain task-representations 


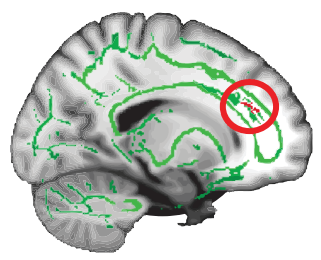

$x=-17$

\section{b AMC seed}

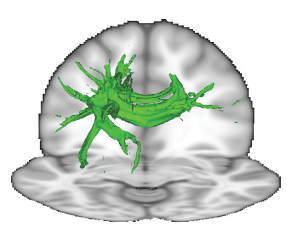

a $A M C$ region

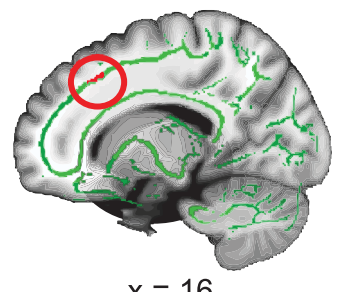

$x=16$

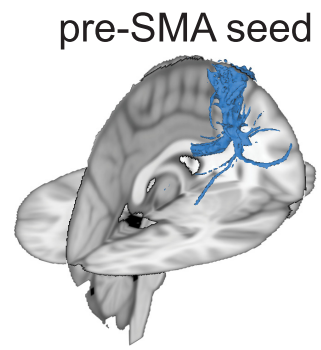

pre-SMA

Figure 4. a, Fractional anisotropy values correlated with post-error slowing in white matter tracts in the area of the anterior midcingulate cortex (AMC) and the pre-SMA. The average WM skeleton is depicted in green. $\boldsymbol{b}$, Probabilistic tractographies. The left anterior midcingulate seed region (AMC seed) is part of fiber tracts connecting medial frontal areas with lateral prefrontal cortex in both hemispheres (probabilistic tractography is depicted in green). Additionally, some tracts connect medial frontal areas with more posterior regions. The right pre-SMA seed region is part of fiber tracts connecting pre-SMA, posterior lateral prefrontal areas, and the subthalamic nucleus (depicted in blue). Pre-SMA is also connected to the homologue area in the left hemisphere.

Table 3. White matter regions where fractional anisotropy values correlated with the individual post-error slowing measure (within average WM skeleton)

\begin{tabular}{lllll}
\hline Region & $x$ & $y$ & $z$ & Volume $\left(\mathrm{mm}^{3}\right)$ \\
\hline WM beneath left anterior midcingulate cortex & -20 & 36 & 25 & 47 \\
WM beneath right pre-SMA & 15 & 26 & 43 & 43 \\
\hline
\end{tabular}

Activation cluster with a threshold of $p<0.001$ and more than 40 contiguously activated voxels are listed.

(Gehring and Knight, 2000; Ullsperger et al., 2002; Ullsperger and von Cramon, 2006) that are strengthened after conflict or errors (Botvinick et al., 2001). Although pMFC and DLPFC activations occur in close temporal proximity, there might be several intermediate neuronal steps between monitoring in pMFC and the updating of task-representations in DLPFC. Therefore, DLPFC activations do not need to be linearly correlated with pMFC activity.

This experiment showed that error-related pMFC activity predicts a subsequent activation decrease in the motor system, which in turn is correlated with the behavioral PES effect. It is thought that PES reflects more controlled responding (Ridderinkhof et al., 2004a). Thus, following errors, it might be a useful strategy that the pMFC not only modulates task-relevant visual areas to enable better performance but also triggers the slowing of the next motor response, which may provide more time for task-focused visual encoding processes. However, activity decreases in motor areas (King et al., 2010) and PES (Notebaert et al., 2009) might be a very general post-error effect that could be functionally independent from activity modulations in visual areas.

Our finding is in line with data from the linear ballistic accumulator model, a mathematical model for exploring speed-accuracy trade-off mechanisms, suggesting that more accurate (posterror) responses occur when response thresholds are higher; ac- cording to this model, the accumulation of decision-relevant information takes time, and thus high response thresholds lead to slower but more accurate responses while low response thresholds lead to faster but more error-prone decisions (Forstmann et al., 2008). In case of post-error trials, lower motor cortex activations might reflect the higher response threshold. Whereas previous evidence for a relationship between $\mathrm{pMFC}$ activity and PES (Gehring et al., 1993; Garavan et al., 2002; Kerns et al., 2004; Debener et al., 2005; di Pellegrino et al., 2007) did not speak to the specific role of the motor system in this adjustment, our finding of reduced post-error motor activity is in line with recent evidence (Marco-Pallares et al., 2008; King et al., 2010) suggesting motor inhibition as a mechanism underlying PES. Consistent with this, transcranial magnetic stimulation (TMS) studies have recently demonstrated that the pre-SMA can modulate M1 activity in conflicting situations and thus influence corticospinal excitability (Mars et al., 2009; Neubert et al., 2010). This corroborates our result showing that pMFC activity predicts the strengths of motor activity following errors, i.e., stronger pMFC activity leads to less motor activity in the post-error trial. Furthermore, a disruption of medial frontal activity by TMS leads to stronger activations of the incorrect response in the motor system in an interference task (Taylor et al., 2007).

To explore whether the PES underlying anatomical structures is consistent with the motor inhibition account, correlations were calculated between PES values and FA values. Several neurobiological factors influence FA values; higher FA values can be a sign of a higher degree of myelination, higher fiber density, bigger axon diameters, or fewer crossing fibers (Beaulieu, 2009; Scholz et al., 2009). Our data revealed that FA values beneath the pMFC, i.e., WM tracts in the vicinity of the right pre-SMA and the left anterior midcingulate cortex, correlated with PES. Tractographies from these seed regions showed that the area beneath the pre-SMA belongs to fiber tracts connecting right-hemispheric pre-SMA, inferior frontal cortex, and the subthalamic nucleus. This network has been associated with stopping and slowing of motor responses (Aron et al., 2007). Consistent with the motor inhibition account of PES, WM integrity of the inhibition network appears to modulate the decrease in motor areas in post-error trials and, therefore PES.

The PES-related left anterior midcingulate area belongs to fiber tracts connecting medial frontal cortical areas with frontopolar and dorsolateral prefrontal cortexes. Frontopolar areas have been associated with attentional dimension weighting (Weidner et al., 2002; Pollmann et al., 2006), i.e., these areas are active when subjects have to shift their attention from one visual feature to another. In the present task, this might reflect an attentional shift away from the motion feature toward the color feature following errors.

In conclusion, by employing combined single-trial fMRI analysis and DWI, this study revealed that error-related pMFC activity drives adaptive processes in visual areas, i.e., a post-error increase in the task-relevant visual area and a peri-error decrease in the task-irrelevant (distractor encoding) visual area. This strongly suggests a top-down regulated attentional shift away from task-irrelevant and toward task-relevant stimulus features. Furthermore, pMFC activity was also related to PES and corresponding adaptations in the motor system. Individual differences in PES were reflected in FA values in WM tracts beneath the pMFC. Motor slowing following errors seems to be triggered by pMFC activity and communicated via an inhibitory network connecting pre-SMA, inferior frontal cortex, and STN. While 
previous work suggested that pMFC activity may be driven in a bottom-up fashion by conflict between task-relevant and distracting inputs (Liston et al., 2006), here we show a top-down influence of pMFC activity biasing motor and visual cortex function in the service of adaptive control.

\section{References}

Aguirre GK, Zarahn E, D’Esposito M (1998) The variability of human, BOLD hemodynamic responses. Neuroimage 8:360-369.

Aron AR, Behrens TE, Smith S, Frank MJ, Poldrack RA (2007) Triangulating a cognitive control network using diffusion-weighted magnetic resonance imaging (MRI) and functional MRI. J Neurosci 27:3743-3752.

Baier B, Kleinschmidt A, Müller NG (2006) Cross-modal processing in early visual and auditory cortices depends on expected statistical relationship of multisensory information. J Neurosci 26:12260-12265.

Beaulieu C (2009) The biological basis of diffusion anisotropy. In: Diffusion MRI: from quantitative measurement to in vivo neuroanatomy (JohansenBerg H, Behrens TEJ, eds). London: Academic.

Behrens TE, Berg HJ, Jbabdi S, Rushworth MF, Woolrich MW (2007) Probabilistic diffusion tractography with multiple fibre orientations: What can we gain? Neuroimage 34:144-155.

Botvinick MM, Braver TS, Barch DM, Carter CS, Cohen JD (2001) Conflict monitoring and cognitive control. Psychol Rev 108:624-652.

Botvinick MM, Cohen JD, Carter CS (2004) Conflict monitoring and anterior

Calhoun VD, Adali T, Pearlson GD, Pekar JJ (2001) A method for making group inferences from functional MRI data using independent component analysis. Hum Brain Mapp 14:140-151.

Carter CS, van Veen V (2007) Anterior cingulate cortex and conflict detection: an update of theory and data. Cogn Affect Behav Neurosci 7:367-379.

Cavanagh JF, Cohen MX, Allen JJ (2009) Prelude to and resolution of an error: EEG phase synchrony reveals cognitive control dynamics during action monitoring. J Neurosci 29:98-105.

Debener S, Ullsperger M, Siegel M, Fiehler K, von Cramon DY, Engel AK (2005) Trial-by-trial coupling of concurrent electroencephalogram and functional magnetic resonance imaging identifies the dynamics of performance monitoring. J Neurosci 25:11730-11737.

di Pellegrino G, Ciaramelli E, Làdavas E (2007) The regulation of cognitive control following rostral anterior cingulate cortex lesion in humans. J Cogn Neurosci 19:275-286.

Egner T, Hirsch J (2005) Cognitive control mechanisms resolve conflict through cortical amplification of task-relevant information. Nat Neurosci 8:1784-1790.

Eichele T, Debener S, Calhoun VD, Specht K, Engel AK, Hugdahl K, von Cramon DY, Ullsperger M (2008) Prediction of human errors by maladaptive changes in event-related brain networks. Proc Natl Acad Sci U S A 105:6173-6178.

Ferrera VP, Lisberger SG (1997) Neuronal responses in visual areas MT and MST during smooth pursuit target selection. J Neurophysiol 78:1433-1446.

Forstmann BU, Dutilh G, Brown S, Neumann J, von Cramon DY, Ridderinkhof KR, Wagenmakers EJ (2008) Striatum and pre-SMA facilitate decision-making under time pressure. Proc Natl Acad Sci USA 105:17538-17542.

Fuster JM (2000) Executive frontal functions. Exp Brain Res 133:66-70.

Garavan H, Ross TJ, Murphy K, Roche RA, Stein EA (2002) Dissociable executive functions in the dynamic control of behavior: inhibition, error detection, and correction. Neuroimage 17:1820-1829.

Gehring WJ, Knight RT (2000) Prefrontal-cingulate interactions in action monitoring. Nat Neurosci 3:516-520.

Gehring WJ, Goss B, Coles MGH, Meyer DE, Donchin E (1993) A neural system for error detection and compensation. Psychol Sci 4:385-390.

Handwerker DA, Ollinger JM, D'Esposito M (2004) Variation of BOLD hemodynamic responses across subjects and brain regions and their effects on statistical analyses. Neuroimage 21:1639-1651.

Himberg J, Hyvärinen A, Esposito F (2004) Validating the independent components of neuroimaging time series via clustering and visualization. Neuroimage 22:1214-1222.

Hyafil A, Summerfield C, Koechlin E (2009) Two mechanisms for task switching in the prefrontal cortex. J Neurosci 29:5135-5142.
Jenkinson M, Smith S (2001) A global optimisation method for robust affine registration of brain images. Med Image Anal 5:143-156.

Jenkinson M, Bannister P, Brady M, Smith S (2002) Improved optimization for the robust and accurate linear registration and motion correction of brain images. Neuroimage 17:825-841.

Kennerley SW, Walton ME, Behrens TE, Buckley MJ, Rushworth MF (2006) Optimal decision making and the anterior cingulate cortex. Nat Neurosci 9:940-947.

Kerns JG, Cohen JD, MacDonald AW 3rd, Cho RY, Stenger VA, Carter CS (2004) Anterior cingulate conflict monitoring and adjustments in control. Science 303:1023-1026.

King JA, Korb FM, von Cramon DY, Ullsperger M (2010) Post-error behavioral adjustments are facilitated by activation and suppression of taskrelevant and task-irrelevant information processing. J Neurosci 30:12759-12769.

Kiviniemi V, Starck, T., Remes, J., Long, X., Nikkinen, J., Haapea, M., Veijola, J., Moilanen, I., Isohanni, M., Zang, Y.F., Tervonen, O. (2009) Functional segmentation of the brain cortex using high model order group PICA. Hum Brain Mapp 30:3865-3886.

Klein TA, Endrass T, Kathmann N, Neumann J, von Cramon DY, Ullsperger M (2007) Neural correlates of error awareness. Neuroimage 34:17741781.

Kouneiher F, Charron S, Koechlin E (2009) Motivation and cognitive control in the human prefrontal cortex. Nat Neurosci 12:939-945.

Liston C, Matalon S, Hare TA, Davidson MC, Casey BJ (2006) Anterior cingulate and posterior parietal cortices are sensitive to dissociable forms of conflict in a task-switching paradigm. Neuron 50:643-653.

Marco-Pallarés J, Camara E, Münte TF, Rodríguez-Fornells A (2008) Neural mechanisms underlying adaptive actions after slips. J Cogn Neurosci 20:1595-1610.

Mars RB, Klein MC, Neubert FX, Olivier E, Buch ER, Boorman ED, Rushworth MF (2009) Short-latency influence of medial frontal cortex on primary motor cortex during action selection under conflict. J Neurosci 29:6926-6931.

Miller BT, D'Esposito M (2005) Searching for "the top" in top-down control. Neuron 48:535-538.

Miller EK, Cohen JD (2001) An integrative theory of prefrontal cortex function. Annu Rev Neurosci 24:167-202.

Müller NG, Kleinschmidt A (2004) The attentional 'spotlight's' penumbra: center-surround modulation in striate cortex. Neuroreport 15:977-980.

Neubert FX, Mars RB, Buch ER, Olivier E, Rushworth MF (2010) Cortical and subcortical interactions during action reprogramming and their related white matter pathways. Proc Natl Acad Sci U S A 107:13240-13245.

Notebaert W, Houtman F, Opstal FV, Gevers W, Fias W, Verguts T (2009) Post-error slowing: an orienting account. Cognition 111:275-279.

Pollmann S, Weidner R, Müller HJ, Maertens M, von Cramon DY (2006) Selective and interactive neural correlates of visual dimension changes and response changes. Neuroimage 30:254-265.

Rabbitt PM (1966) Errors and error correction in choice-response tasks. J Exp Psychol 71:264-272.

Ridderinkhof KR, van den Wildenberg WPM, Wijnen J, Burle B (2004a) Response inhibition in conflict tasks is revealed in delta plots. In: Cognitive neuroscience of attention (Posner MI, ed). New York: Guilford.

Ridderinkhof KR, Ullsperger M, Crone EA, Nieuwenhuis S (2004b) The role of the medial frontal cortex in cognitive control. Science 306:443-447.

Sarter M, Gehring WJ, Kozak R (2006) More attention must be paid: the neurobiology of attentional effort. Brain Res Rev 51:145-160.

Scholz J, Tomassini V, Johansen-Berg H (2009) Individual differences in white matter microstructure in the healthy brain. In Diffusion MRI: from quantitative measurement to in vivo neuroanatomy (Johansen-Berg $\mathrm{H}$, Behrens TEJ, eds). London: Academic.

Simon JR (1969) Reactions toward source of stimulation. J Exp Psychol $81: 174-176$.

Smith SM (2002) Fast robust automated brain extraction. Hum Brain Mapp 17:143-155.

Smith SM, Jenkinson M, Woolrich MW, Beckmann CF, Behrens TE, Johansen-Berg H, Bannister PR, De Luca M, Drobnjak I, Flitney DE, Niazy RK, Saunders J, Vickers J, Zhang Y, De Stefano N, Brady JM, Matthews PM (2004) Advances in functional and structural MR im- 
age analysis and implementation as FSL. Neuroimage 23 [Suppl 1]:S208-S219.

Smith SM, Jenkinson M, Johansen-Berg H, Rueckert D, Nichols TE, Mackay CE, Watkins KE, Ciccarelli O, Cader MZ, Matthews PM, Behrens TE (2006) Tract-based spatial statistics: voxelwise analysis of multi-subject diffusion data. Neuroimage 31:1487-1505.

Smith SM, Johansen-Berg H, Jenkinson M, Rueckert D, Nichols TE, Miller KL, Robson MD, Jones DK, Klein JC, Bartsch AJ, Behrens TE (2007) Acquisition and voxelwise analysis of multi-subject diffusion data with tract-based spatial statistics. Nat Protoc 2:499-503.

Taylor PC, Nobre AC, Rushworth MF (2007) Subsecond changes in top down control exerted by human medial frontal cortex during conflict and action selection: a combined transcranial magnetic stimulation electroencephalography study. J Neurosci 27:11343-11353.

Teufel HJ, Wehrhahn C (2000) Evidence for the contribution of S cones to the detection of flicker brightness and red-green. J Optic Soc Am A 17:994-1006.

Treue S (2001) Neural correlates of attention in primate visual cortex. Trends Neurosci 24:295-300.

Ullsperger M, von Cramon DY (2001) Subprocesses of performance monitoring: a dissociation of error processing and response competition revealed by event-related fMRI and ERPs. Neuroimage 14:13871401.

Ullsperger M, von Cramon DY (2006) The role of intact frontostriatal circuits in error processing. J Cogn Neurosci 18:651-664.

Ullsperger M, von Cramon DY, Müller NG (2002) Interactions of focal cortical lesions with error processing: evidence from event-related brain potentials. Neuropsychology 16:548-561.

Vanduffel W, Tootell RB, Orban GA (2000) Attention-dependent suppression of metabolic activity in the early stages of the macaque visual system. Cereb Cortex 10:109-126.

Weidner R, Pollmann S, Müller HJ, von Cramon DY (2002) Top-down controlled visual dimension weighting: an event-related fMRI study. Cereb Cortex 12:318-328.

Woolrich MW, Ripley BD, Brady M, Smith SM (2001) Temporal autocorrelation in univariate linear modeling of FMRI data. Neuroimage 14:1370-1386.

Wylie GR, Javitt DC, Foxe JJ (2006) Jumping the gun: is effective preparation contingent upon anticipatory activation in task-relevant neural circuitry? Cereb Cortex 16:394-404. 